\title{
Separating Astrophysics and Geometry in Black Hole Images
}

\author{
Guillermo Lara, ${ }^{*}$ Sebastian H. Völkel ${ }^{\dagger}$ and Enrico Barausse \\ SISSA - Scuola Internazionale Superiore di Studi Avanzati, \\ via Bonomea 265, 34136 Trieste, Italy and INFN Sezione di Trieste and \\ IFPU - Institute for Fundamental Physics of the Universe, via Beirut 2, 34014 Trieste, Italy
}

(Dated: December 20, 2021)

\begin{abstract}
The observation of the shadow of the supermassive black hole M87* by the Event Horizon Telescope (EHT) is sensitive to the spacetime geometry near the circular photon orbit and beyond, and it thus has the potential to test general relativity in the strong field regime. Obstacles to this program, however, include degeneracies between putative deviations from general relativity and both the description of the accretion flow and the uncertainties on "calibration parameters", such as e.g. the mass and spin of the black hole. In this work, we introduce a formalism, based on a principal component analysis, capable of reconstructing the black hole metric (i.e. the "signal") in an agnostic way, while subtracting the "foreground" due to the uncertainties in the calibration parameters and the modelling of the accretion flow. We apply our technique to simulated mock data for spherically symmetric black holes surrounded by a thick accretion disk. We show that separation of signal and foreground may be possible with next generation EHT-like experiments.
\end{abstract}

\section{INTRODUCTION}

More than a century after Albert Einstein introduced general relativity (GR) [1], many large scale observational campaigns and theoretical studies are still searching for possible, new insights into gravity. Although GR has so far passed all tests with flying colors, e.g., in recent years the exploration of the dynamical and strong field regime via measurements of black hole $(\mathrm{BH})$ and neutron star mergers by LIGO and Virgo [2-5], many of the outstanding open problems in physics involve gravitation and BHs. The latter are among the most extreme objects in nature, and access to the gravitational waves that they emit, as well as the first radio "image" of the shadow of the supermassive BH M87* by the Event Horizon Telescope (EHT) [6, 7], allow for testing our theoretical understanding in unprecedented ways.

One possible way to look for deviations from GR is to test the Kerr hypothesis [8-10]. The latter states that all astrophysical BHs are uniquely characterized by their mass and angular momentum through the Kerr metric [11]. The recent measurement of the shadow of M87* is therefore particularly interesting to test the Kerr hypothesis and constrain possible deviations from it. Several theoretical works have connected the bright emission ring that is present in the reconstructed image with the impact parameter of the circular photon orbit [1225]. This feature in the image may therefore be used to connect the underlying $\mathrm{BH}$ spacetime with the observed image and therefore test the Kerr hypothesis.

Despite this large amount of existing theoretical work on the topic, a lively discussion about the interpretation of $\mathrm{BH}$ shadow images is currently ongoing. More precisely, one question is how suited the shadow size alone

\footnotetext{
* jlaradel@sissa.it

$\dagger$ svoelkel@sissa.it

$\ddagger$ barausse@sissa.it
}

(as opposed to the full image) is for testing deviations from the Kerr metric. In Psaltis et al. [26], it was claimed that the $17 \%$ constraint on the shadow size of M87*, as reported earlier by the EHT Collaboration in Refs. [6, 7], can be used to place constraints beyond the first postNewtonian order of the BH metric. However, the basis and robustness of this conclusion have been critically discussed since then. Gralla [27] argued that uncertainties in the underlying astrophysics make tests of GR with the current observations not possible. Völkel et al. [28] showed that even if some underlying assumptions questioned by Gralla [27] and adopted in Psaltis et al. [26] are true, the bounds of Ref. [26] do not hold when higher order post-Newtonian orders are included in the analysis. Nevertheless, Völkel et al. [28], as well as Kocherlakota et al. [29], demonstrate that theory specific tests can be performed and allow for gaining information on the gravitational theory. The role of dimensional coupling constants in alternative theories of gravity has been studied by Glampedakis and Pappas [30].

Since the shadow size, as used in most previous works ${ }^{1}$, is only a single number, it represents an immense reduction of the information that is encoded in the full image. For this reason, and because it is based on the connection between the impact parameter of the circular photon orbit and the image brightness, more advanced studies are needed.

Improvements of several aspects of the analysis have already been partially carried out in recent works. For instance, a multiple ring structure seems to appear in the shadow image at high angular resolution, although its observation would require measurements beyond current EHT capabilities, see e.g. Refs. [32-34]. Medeiros et al. [24] have also investigated deviations from circularity in non-Kerr spacetimes using a principal component

\footnotetext{
1 See Nampalliwar et al. [31] for a recent work including also the deviations from circularity.
} 
analysis (PCA). Formal questions regarding the uniqueness of shadow images and the underlying BH geometry, from the point of view of geodesics, have been recently studied in Ref. [35].

Ideally, however, an analysis should take into account the whole image and simulate both the astrophysics and the background geometry at the same time. Treating this problem in its full complexity is far beyond of what is currently possible. State-of-the-art calculations combine general relativistic magneto-hydrodynamic (GRMHD) simulations of the matter with ray-tracing codes to construct images -see Ref. [7, 36]. However, even in the context of GR, the large computational cost associated to these calculations allows one to vary (at the same time) at most a few parameters describing the accretion flow physics and the geometry [36, 37]. Therefore, repeating these simulations for non-Kerr spacetimes is already challenging, even when backreaction on the geometry is neglected -see e.g. Refs. [38, 39]. Nevertheless, varying the accretion flow physics and allowing for nonKerr geometries at the same time has been attempted before, although restricting to particular deformations of the metric [40].

In this work, we present a framework that allows one to deal with deviations of both the accretion flow and the spacetime geometry from standard scenarios, showing that under suitable assumptions they can be both recovered/constrained from the shadow image. In more detail, we perform a PCA, which allows us to probe small but very general (i.e. theory "agnostic") deviations from the Schwarzschild metric, while at the same time reconstructing the astrophysical accretion flow (which we assume to be spherical and with a simple emissivity profile). The PCA allows for working in a rather general set of basis functions, e.g. Gaussians or power laws. Although our matter description is not as sophisticated as current GRMHD simulations, we show for the first time how the background metric and simple astrophysical scenarios can be disentangled from one another and reconstructed singularly in an inverse problem for the whole shadow image. Our work is therefore extensible to incorporate more realistic models in the future.

Our main findings can be summarized as follows. We provide different mock images produced by our model as hypothetically observed data. These images have been produced by varying the emissivity profile and/or the $\mathrm{BH}$ metric. We have first demonstrated that the PCA reconstruction allows one to constrain the emissivity profile when the background is assumed to be Schwarzschild. Then we have also demonstrated that general deviations of the metric away from Schwarzschild can be recovered when the emissivity profile is well understood. In the final and most important application, we have demonstrated that when the functional form of the emissivity profile is assumed to be of a simple form, but with unknown parameters, it is possible to recover both the spacetime geometry (the "signal") in an agnostic way, as well as the emissivity profile (the "matter foreground").
This work is organized as follows. In Sec. II we summarize our accretion model and the ray-tracing algorithm. In Sec. III we outline our numerical method to compute $\mathrm{BH}$ images and the PCA. Applications and results are reported in Sec. IV. A discussion of our findings is presented in Sec. V, while our final conclusions are provided in Sec. VI. The metric signature throughout this paper is -+++ and we set $G=c=1$.

\section{THEORY}

In the following, we will give a brief overview of our modelling of accretion flows (in Sec. II A) and of raytracing around supermassive BHs (in Sec. II B).

\section{A. Accretion model}

Being a bright source for terrestrial and space observatories across different parts of the electromagnetic spectrum, M87* has been extensively observed in a variety of wavelengths [41]. The central $\mathrm{BH}$ mass can be estimated by modeling the dynamics of nearby gas [42] or stars [43]. Note that these methods give different $\mathrm{BH}$ mass values, but the recent EHT measurement of $M_{\mathrm{BH}}=(6.5 \pm 0.7) \times 10^{9} M_{\odot}$ only agrees with the stellar dynamics measurement [7]. The environment of M87* is believed to be a geometrically thick and optically thin accretion disk [36], rather than a geometrically thin and optically thick one (such as e.g. the classic NovikovThorne model [44]). The photons observed by the EHT at $1.3 \mathrm{~mm}$ are thought to be produced by synchrotron radiation from the relativistic electrons in the hot accretion plasma $[6,36]$. Moreover, M87* exhibits a visible jet, observable at all wavelengths, whose power has been used to disfavor zero-spin models [36, 45].

For our purposes, we employ a simplified toy model for the accretion flow. We assume a spherically symmetric and optically thin disk surrounding a spherically symmetric (i.e. non-rotating) $\mathrm{BH}$ with metric

$$
d s^{2}=g_{t t}(r) d t^{2}+g_{r r}(r) d r^{2}+r^{2} d \theta^{2}+r^{2} \sin ^{2}(\theta) d \phi^{2},
$$

where $g_{t t}(r) g_{r r}(r)=-B(r)^{2}$, with $B(r)$ being a free function. The power radiated by the disk can be characterized by the emissivity [46],

$$
j_{\nu}(r)=\frac{d E}{d V d t d \nu},
$$

which we assume to be independent of the frequency $\nu$. Motivated by radiatively inefficient accretion flow (RIAF) models [40, 47], we assume that the spatial distribution of the emissivity is well-described by a power law $j_{\nu}(r) \propto r^{-n}$, with $n \approx 1$.

We assume that the $\mathrm{BH}$ image is detected by a distant observer. Hence, the image can be described by the intensity $I_{\nu}(b)$, as a function of the impact parameter $b$ of the 
null geodesics along which photons propagate. The intensity can be obtained by integrating the radiative transfer equations (here presented in the form of Ref. [48, 49]),

$$
\begin{aligned}
\frac{d}{d \lambda}\left(\frac{I_{\nu, \mathrm{obs}}}{\nu_{\mathrm{obs}}^{3}}\right) & =\frac{j_{\nu}}{\nu^{2}} e^{-\tau_{\nu, \mathrm{obs}}}, \\
\frac{d \tau_{\nu, \mathrm{obs}}}{d \lambda} & =\alpha_{\nu} \nu
\end{aligned}
$$

where $\nu=-\kappa_{\mu} u^{\mu}$, with $\kappa^{\mu}$ the photon's linear momentum and $u^{\mu}$ the 4 -velocity of the disk's fluid, is the photon's frequency as measured in the fluid's rest frame. The subscript "obs" denotes quantities in the observer's frame.

These equations are integrated from the matter source to the far away observer along null geodesics parametrized by the affine parameter $\lambda$. Since we make the assumption of a disk that is (perfectly) optically thin, we choose an absorption coefficient $\alpha_{\nu}=0$, and we also neglect the Doppler shift due to the motion of the disk's fluid, i.e. we assume $u^{\mu}=\left(1 / \sqrt{-g_{t t}}, 0,0,0\right)$. Furthermore, we assume that the observer measures $I_{\nu, \mathrm{obs}}(b)$ at a single frequency. ${ }^{2}$ Hereinafter, for simplicity, we will drop the labels $\nu$ and "obs" in both the intensity and the emissivity.

\section{B. Ray-tracing}

The photons contributing to the $\mathrm{BH}$ image probe the spacetime along null geodesics. In the vicinity of a Schwarzschild BH, these photons are strongly lensed. For a critical impact parameter $b_{\mathrm{ph}}=3 \sqrt{3} M_{\mathrm{BH}}$, where $M_{\mathrm{BH}}$ is the mass of the $\mathrm{BH}$, photons follow unstable circular orbits at a surface called the photon sphere. If no matter is present within this surface, this critical value of $b_{\mathrm{ph}}$ can be interpreted as the size of the $\mathrm{BH}$ shadow. [50]

In practice, although photons follow trajectories from the source to the observer, ray-tracing algorithms often integrate the geodesics and radiative transfer equations in the opposite direction - see e.g. Ref. [49]. In addition, although the geodesics equations are integrable in spherical symmetry (and in the Kerr spacetime), it is convenient to consider the standard second-order equations

$$
\frac{d^{2} \kappa^{\mu}}{d \lambda^{2}}+\Gamma_{\rho \sigma}^{\mu} \frac{d \kappa^{\rho}}{d \lambda} \frac{d \kappa^{\sigma}}{d \lambda}=0,
$$

where $\Gamma_{\rho \sigma}^{\mu}$ are the Christoffel symbols. The advantages of this choice are that one avoids a special treatment at the turning points of the geodesics, and (as we will describe below) one can linearly perturb these equations in a straightforward way.

\footnotetext{
2 In practice, the EHT measures visibilities, which correspond to the Fourier transform coefficients of the Stokes parameters of the image.
}

\section{METHODS}

In this Section, we outline our PCA method. In more detail, in Sec. III A we introduce the basis functions on which we decompose the accretion model and the deviations of the BH metric away from Schwarzschild. In Sec. III B, we present our (linearized) likelihood, and apply the PCA to it in Sec. III C. The role of priors is discussed in Sec. IIID.

\section{A. Choice of basis}

A useful way to describe and test the multiple possible modified gravity solutions for $\mathrm{BH}$ geometries is to employ physically motivated parametrizations of the metric. In spherical symmetry, these can take the form of series of $r^{-n}$ terms, with $n \neq 0$, like in the postNewtonian (PN) series, or more complicated expressions, like in the Rezzolla-Zhidenko parametrization, where the metric coefficients are described by Padé approximants $[51,52]$. One must be careful, however, that any particular parametrization may not be able to describe all possible arbitrary departures from GR, especially if only few parameters are left free to vary. ${ }^{3}$ For instance, the PN expansion is valid only for mild gravitational fields, c.f. for instance Ref. [28], and an infinite number of terms would be needed to describe all possible conceivable $\mathrm{BH}$ metrics in the strong field region.

In the following, we therefore allow the deviations of the metric from the Schwarzschild solution to have an arbitrary form. Focusing on $g_{t t}(r)$ and assuming for simplicity a toy model where $B=1$ (and hence $\left.g_{t t}(r) g_{r r}(r)=-1\right)$, one can write

$$
g_{t t}(r)=g_{t t}^{(0)}(r)+\sum_{i} \alpha_{i}^{(t t)} \delta g_{t t}^{(i)}(r),
$$

where $\alpha_{i}^{(t t)}$ are free coefficients, $g_{t t}^{(0)}(r)$ is the Schwarzschild solution, and the functions $\delta g_{t t}^{(i)}(r)$ form a suitable basis on which any smooth function defined on the (positive) real axis can be decomposed. The basis is sometimes referred to also as frame. A familiar example of basis functions is given by sines and cosines in Fourier analysis. Another example is provided by the Morlet-Gabor wavelets used in gravitational wave analysis pipelines [54]. This basis need not be orthogonal. For instance, in some of the applications described in the following, we choose the basis to consist of Gaussians (centered on different points on the real axis, labelled by

\footnotetext{
3 Note however that the Rezzolla-Zhidenko parametrization has proven very flexible in this respect, as it is capable of reproducing $\mathrm{BH}$ geometries in entire classes of theories [53] with relatively few parameters. However, those theories are not necessarily comprehensive of all possible deviations from GR that one can conceive.
} 
a discrete index $i$, but with a non-vanishing overlap to enforce continuity of the reconstructed function).

In order to account for uncertainties in the astrophysical model, we can write a similar expression for the emissivity of the disk:

$$
j(r)=j^{(0)}(r)+\sum_{i} \alpha_{i}^{(J)} \delta j^{(i)}(r)
$$

where $\alpha_{i}^{(J)}$ are again free coefficients, and $\delta j^{(i)}(r)$ may be different from the metric basis functions.

\section{B. Linearized Model and Likelihood}

We approach the inverse problem of reconstructing the spacetime metric from the observed $\mathrm{BH}$ image within a Bayesian perspective. The expressions in Eqs. (6) and (7) will be the backbone of our model $I_{M}(\alpha, b)$ for the $\mathrm{BH}$ image. We will then seek to estimate the parameters $\boldsymbol{\alpha}=\left(\alpha_{l}^{(t t)}, \alpha_{m}^{(J)}\right)$ that best describe the $\mathrm{BH}$ image data $I_{D}(b)$. When the posterior probability can be approximated as Gaussian, one can further "clean" the reconstructed metric by means of a PCA.

We begin by describing our model for the $\mathrm{BH}$ image in more detail. We assume that the image consists of a finite number of data points at locations $b_{1}, \ldots, b_{N}$, where the associated intensity $\boldsymbol{I}_{D}=\left(I_{D, 1}, \ldots, I_{D, N}\right)$ is measured. We also assume that the data are subject to Gaussian measurement errors, which we assume to be constant and given by $\sigma$. As for our model, which we denote by $\boldsymbol{I}_{M}(\boldsymbol{\alpha})=\left(I_{M}\left(\boldsymbol{\alpha}, b_{1}\right), \ldots, I_{M}\left(\boldsymbol{\alpha}, b_{N}\right)\right)$, we integrate numerically the radiative transfer and geodesic equations [Eqs. (3) and (5)] assuming Eqs. (6) and (7). In particular, in order to render the posterior probability function Gaussian (i.e. quadratic in the parameters $\boldsymbol{\alpha}$ ) and apply the PCA technique, we linearize Eqs. (3) and (5) in $\boldsymbol{\alpha}$.

Physically, this amounts to assuming that the deviations from Schwarzschild and from our default emissivity model are small. (We do not report these linearized equations here as they are cumbersome and not particularly illuminating - see e.g. Appendix A of Ref. [55] for the linear perturbations of the geodesic equations.) In practice, the 14 first-order linearized equations for the variables

$$
\left\{t, r, \phi, \dot{t}, \dot{r}, \dot{\phi}, \delta t, \delta r, \delta \phi, \delta \dot{t}, \delta \dot{r}, \delta \dot{\phi}, I^{(0)}, \delta I\right\},
$$

where $\dot{X} \equiv d X / d \lambda$, are integrated with a custom-made ray-tracing code written in $\mathrm{C}++$ and employing an adaptive stepsize fourth order Runge-Kutta algorithm [56]. Therefore, the model can be written as

$$
\boldsymbol{I}_{M}(\boldsymbol{\alpha})=\boldsymbol{I}^{(0)}+\sum_{i=1}^{M} \alpha_{i} \delta \boldsymbol{I},
$$

where $M$ is the total number of $\alpha_{i}$ parameters and $\delta \boldsymbol{I}$ are the image deviations corresponding to each of the individual basis functions. ${ }^{4}$

In the Bayesian framework, the solution to the inverse problem, up to a normalization factor, is encoded in the posterior probability distribution, given by

$$
p\left(\boldsymbol{\alpha} \mid \boldsymbol{I}_{D}\right) \propto p\left(\boldsymbol{I}_{D} \mid \boldsymbol{\alpha}\right) p(\boldsymbol{\alpha}),
$$

where the likelihood follows from the assumption of Gaussian measurement errors and is given by

$$
\log p\left(\boldsymbol{I}_{D} \mid \boldsymbol{\alpha}\right)=-\frac{\chi^{2}}{2}
$$

with

$$
\chi^{2}=\frac{1}{\sigma^{2}}\left(\boldsymbol{I}_{D}-\boldsymbol{I}_{M}(\boldsymbol{\alpha})\right)^{T}\left(\boldsymbol{I}_{D}-\boldsymbol{I}_{M}(\boldsymbol{\alpha})\right) .
$$

In addition, as explained below, we will assume Gaussian priors $p(\boldsymbol{\alpha})$. This choice, coupled with the likelihood Eq. (12), yields Gaussian posteriors, which are suitable for PCA.

\section{Principal Component Analysis}

Since the model is linear in the parameters and the posterior probability is Gaussian, the maximum of the latter (i.e. the "most likely" parameters $\boldsymbol{\alpha}^{\star}$ ) can be obtained by solving a (possibly degenerate) linear system of equations of the form $\boldsymbol{F} \boldsymbol{\alpha}+\boldsymbol{q}=0$, where the $M \times M$ matrix $\boldsymbol{F}$ (defined below) and the $M$-vector $\boldsymbol{q}$ are computed numerically with our ray-tracing code. (This is the most computationally expensive part of the framework since it involves computing a perturbed image for every basis function.) The errors associated with the parameters are in general correlated and are encoded in the Fisher (Hessian) matrix

$$
F_{l m}=-\frac{1}{2} \frac{\partial^{2}}{\partial \alpha_{l} \partial \alpha_{m}} \log p\left(\boldsymbol{\alpha} \mid \boldsymbol{I}_{D}\right),
$$

which, with our assumptions, becomes a constant matrix. Linear combinations of the parameters corresponding to the Fisher matrix orthonormal eigenvectors $\boldsymbol{e}^{(i)}$, however, have uncorrelated errors $\sigma^{(i)}=1 / \sqrt{2 \lambda^{(i)}}$, where $\lambda^{(i)}$ are the corresponding eigenvalues.

The main idea behind the PCA is to clean the reconstruction of the model by keeping only the "largest" coefficients $\beta_{i}=\boldsymbol{\alpha}^{\star} \cdot \boldsymbol{e}^{(i)}$. This is akin to the procedure of cleaning a time series from noise by performing a Fourier transform, and then keeping only the Fourier terms with coefficients significantly different from zero.

\footnotetext{
${ }^{4}$ Notice that the basis functions (dependent on the coordinate variable $r$ ) are mapped into an image space (dependent on the impact parameter variable $b$ ) by a nonlinear transformation or "transfer function". In many applications of the PCA (e.g. Ref. [57]) there is no such mapping.
} 
More precisely, we prescribe the selection criterion [57]

$$
\left|\beta_{i}\right| \geq N_{\mathrm{th}} \sigma^{(i)}
$$

whereby we only retain the coefficients $\beta_{i}$ that are inconsistent with zero at $N_{\mathrm{th}}$-th sigma level, with $N_{\mathrm{th}} \approx 1-3$. The final estimate of the parameters is then given by

$$
\boldsymbol{\alpha}^{\mathrm{PCA}} \equiv \sum_{\left|\beta_{i}\right| \geq N_{\mathrm{th}} \sigma^{(i)}}\left(\beta_{i} \pm \sigma^{(i)}\right) \boldsymbol{e}^{(i)} .
$$

Explicitly, the reconstructed metric and emissivity are then given by

$$
\begin{aligned}
& g_{t t}^{\mathrm{PCA}}(r) \equiv g_{t t}^{(0)}(r)+\sum_{\left|\beta_{i}\right| \geq N_{\mathrm{th}} \sigma^{(i)}}\left(\beta_{i} \pm \sigma^{(i)}\right) \eta^{(i),(t t)}(r), \\
& j^{\mathrm{PCA}}(r) \equiv j^{(0)}(r)+\sum_{\left|\beta_{i}\right| \geq N_{\mathrm{th}} \sigma^{(i)}}\left(\beta_{i} \pm \sigma^{(i)}\right) \eta^{(i),(J)}(r),
\end{aligned}
$$

where

$$
\begin{aligned}
\eta^{(i),(t t)}(r) & \equiv \sum_{k} e_{k}^{(i),(t t)} \delta g_{t t}^{(k)}(r), \\
\eta^{(i),(J)}(r) & \equiv \sum_{k} e_{k}^{(i),(J)} \delta j^{(k)}(r),
\end{aligned}
$$

are the corresponding eigenfunctions. Here we have separated the eigenvectors $\boldsymbol{e}^{(i)}=\left(e_{l}^{(i),(t t)}, e_{m}^{(i),(J)}\right)$ according to their corresponding metric and emissivity indices. Note that because the coefficients $\beta_{i}$ are uncorrelated Gaussian variables, the errors on the reconstructed metric and emissivity at a given location can be computed by propagating the errors $\sigma^{(i)}$ in quadrature. These errors will be reported in Sec. IV.

\section{Priors}

The use of priors in the reconstruction can enhance it by mitigating some of the degeneracies that may be present. First, the linear system needed to solve for $\boldsymbol{\alpha}^{\star}$, and thus to obtain $\boldsymbol{\alpha}^{\mathrm{PCA}}$, is in general degenerate. One way to tame this issue is by conditioning the Fisher matrix $F_{l m}$, i.e. by replacing $F_{l m} \rightarrow F_{l m}+\epsilon \delta_{l m}$, where $\delta_{l m}$ is the Kronecker delta and $\epsilon$ is suitably small [57]. From the Bayesian perspective, this can be interpreted as prescribing loose Gaussian priors for $\boldsymbol{\alpha}$ centered at zero. At least in the case of the metric, this is in line with the theoretical expectation that the parameters $\alpha_{i}^{(t t)}$, which describe the deviations from GR, should be small.

Proper priors on the coefficients of neighboring basis functions can also be used to enforce continuity [58]. More importantly, from a physical point of view, priors can be used to enforce the expectation that the metric deviations are well constrained at large distances, where the Newtonian limit of GR (but not necessarily its 1PN dynamics [28]) should be recovered. For the Gaussian basis functions uniformly distributed in radius that we will use below, we therefore include a prior of the form

$$
p(\alpha) \propto \exp \left(-\left(\frac{r_{(i)}}{M_{\mathrm{BH}}}\right)^{n} \sum_{i} \frac{\alpha_{i}^{(t t) 2}}{2 \sigma_{r}^{2}}\right),
$$

with $n=4$, constant $\sigma_{r}$ and $r_{(i)}$ the location of the Gaussian basis function associated to $\alpha_{i}^{(t t)}$. Equivalently, this corresponds to the expectation that the deviations from GR enter at 1PN order or higher [28]. This prior has also the advantage that it stabilizes the reconstruction against fluctuations from different noise realizations. Finally, the choice of the reference functions $g_{t t}^{(0)}(r)$ and $j^{(0)}(r)$ constitutes an additional "theoretical prior".

\section{APPLICATIONS AND RESULTS}

We apply our framework to the following cases. First, in Sec. IV A we assume that the spacetime geometry is known and only the accretion flow, described by the emissivity, needs to be reconstructed. Second, in Sec. IV B we consider the opposite case in which the emissivity is assumed to be known, but the spacetime geometry needs to be reconstructed. Finally, in Sec. IV C we let both the spacetime geometry and the accretion flow model undetermined at the same time. In order to directly compare the results obtained in these three ways, we show the corresponding reconstructions in the same figures at the end of this Section. Since we do not realistically model the details of the noise in the simulated observations, in the following we will focus on injections in the noiseless approximation. This for instance standard when designing gravitational wave data analysis pipelines [59]. However, we have checked that the results are similar for explicit realizations of the Gaussian noise.

In the following, we also introduce the scales $j_{*}$ and $I_{*}$, with units $\left[j_{*}\right]=[$ energy $][\text { length }]^{-3}$ and $\left[I_{*}\right]=$ [length] $\left[j_{*}\right]$, as a normalizing scales for the emissivity and observed intensity.

\section{A. Reconstructing the Accretion Flow}

We fix the reference metric function to be that of the Schwarzschild geometry, $g_{t t}^{(0)}(r)=-\left(1-2 M_{\mathrm{BH}} / r\right)$, without allowing for any deviations from it -i.e. only the astrophysical parameters $\alpha_{i}^{(J)}$ are allowed to vary. As for the reference emissivity, we choose it to be $j^{(0)}(r)=0$ (i.e. we assume no prior knowledge of the emissivity). We produce the injection (i.e. the data) $\boldsymbol{I}_{D}$ from

$$
g_{t t, D}(r)=g_{t t}^{(0)}(r), \quad j_{D}(r)=2 j_{*} \frac{M_{\mathrm{BH}}}{r} .
$$

We assume an optimistic resolution of $0.15 M_{\mathrm{BH}}$ (potentially achievable with future space-based interferometers 
[60]). More precisely, we generate $N=100$ data points uniformly distributed in the interval of impact parameters $\left[0,15 M_{\mathrm{BH}}\right]$, and assume an (uncorrelated) measurement error of $\sigma=0.1 I_{*}$. The basis is composed of 161 (unnormalized) Gaussians uniformly distributed in the interval of radii $\left[0,100 M_{\mathrm{BH}}\right]$, with root mean square (RMS) width of $1 M_{\mathrm{BH}}$. We condition the Fisher matrix with $\epsilon=1$, which can also be interpreted as a Gaussian prior, as discussed in Sec. IIID.

In the upper panel of Fig. 1, in blue, we show the intensity profile for the injection Eq. (21), as a function of the impact parameter. One can clearly observe the effect of the photon sphere at $b \approx 5.2 M_{\mathrm{BH}}$. In Fig. 2, with blue contours, we show the $2 \sigma$ bands of the reconstructed emissivity profile for a PCA criterion parameter $N_{\text {th }}=1$. We observe good agreement with the injection (gray dashed lines) for most radii. The reconstruction, however, does not accurately reproduce the emissivity profile near the $\mathrm{BH}$ horizon at $r=2 M_{\mathrm{BH}}$. This disagreement is most likely due to the effect of gravitational redshift, which suppresses the intensity of the near-horizon Gaussian components. The widening of the reconstruction bands at $r \approx 14 M_{\mathrm{BH}}$ occurs instead because the image data were provided in a finite interval -i.e. it is an "edge effect".

\section{B. Reconstructing the Geometry}

In the following, we present the results of our framework when the accretion flow is assumed to be known and only the background metric is reconstructed -i.e. when only the parameters $\alpha_{i}^{(t t)}$ are allowed to vary.

For this example, the injection includes a deviation from GR in the metric, which is given by

$$
\begin{gathered}
g_{t t, D}(r)=g_{t t}^{(0)}(r)-0.02 \exp \left[-\frac{\left(r-7 M_{\mathrm{BH}}\right)^{2}}{M_{\mathrm{BH}}^{2}}\right]+ \\
\quad-0.8\left(\frac{M_{\mathrm{BH}}}{r}\right)^{3} \\
j_{D}(r)=j^{(0)}(r)=2 j_{*} \frac{M_{\mathrm{BH}}}{r}
\end{gathered}
$$

where $g_{t t}^{(0)}(r)=-\left(1-2 M_{\mathrm{BH}} / r\right)$. For the sake of simplicity, we also set the reference function for the emissivity to be the same as the injection -although we could have chosen a slightly different profile without strongly affecting our results. The resolution and measurement errors are the same as in the previous example. We choose a basis of 161 Gaussians uniformly distributed in the interval $\left[0,25 M_{\mathrm{BH}}\right]$ and with RMS width of $0.5 M_{\mathrm{BH}}$, supplemented by additional power law components $r^{-n}$, with $n=1,2, \ldots, 15$. To improve the conditioning of the Fisher matrix, we also rescale the coefficients $\boldsymbol{\alpha}$ so that the different entries in that matrix are roughly of the same order of magnitude. The $r^{-1}$ power law accounts for the uncertainty in our knowledge of the mass,

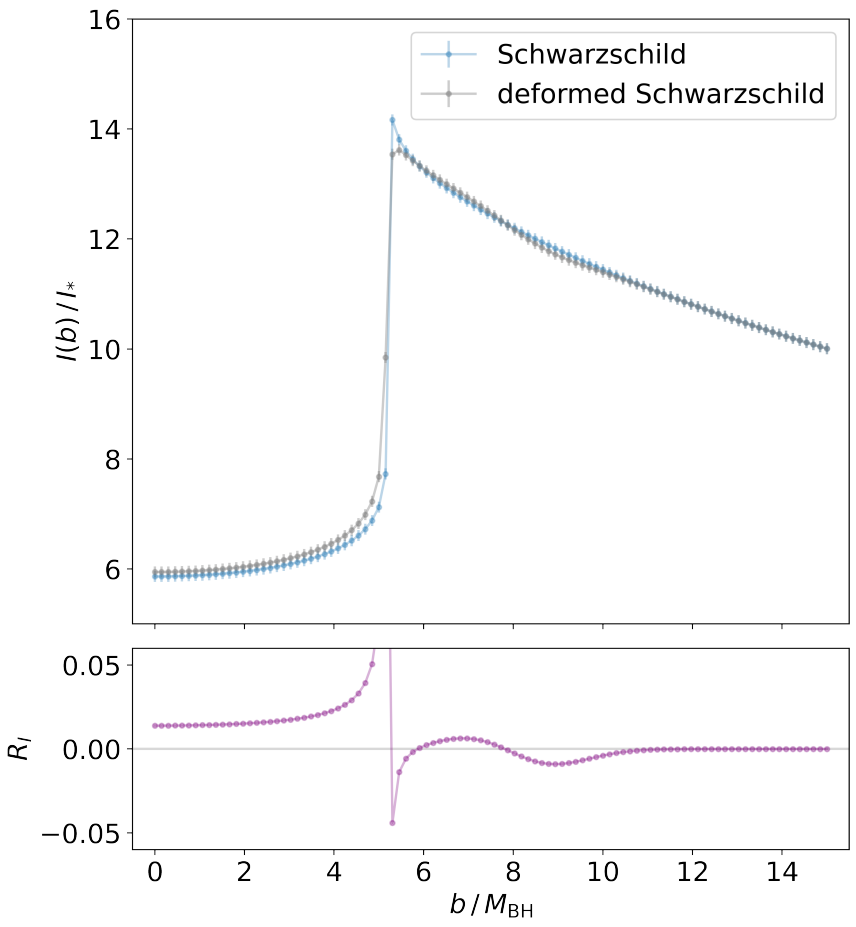

FIG. 1. Upper panel: Computed intensity profiles $I(b)$ used as "observations", as a function of the impact parameter $b$ for the Schwarzschild metric (blue) and the deformed Schwarzschild metric (gray), with corresponding "measurement" error bars of $\pm 0.1 I_{*}$. The scale $I_{*}$ is used to normalize the intensity profile, and its units are given in the main text. The Schwarzschild intensity profile is used as input data for the example of Sec. IV A, while the deformed Schwarzschild one is used in the examples of Secs. IV B and IV C. Lower panel: Relative difference $R_{I}=\left(I_{\mathrm{Sch}}-I_{\mathrm{def}-\mathrm{Sch}}\right) / I_{\mathrm{Sch}}$ of the images in the upper panel.

for which we assume a small prior measurement error of $\sigma_{M_{\mathrm{BH}}}=0.01 M_{\mathrm{BH}}$. Finally, we take $\epsilon=10^{-3}$ for the conditioning of the Fisher matrix, and $\sigma_{r}^{2}=10^{5}$ for the large distance prior of Eq. (20).

The intensity profile used as data and produced from Eq. (22) is shown in the upper panel of Fig. 1, in gray. Here, one can observe the breakdown of the linear approximation to the full geodesics equation at $b \approx 5.2 M_{\mathrm{BH}}$, where the linear approximation fails to reproduce the shift in the photon sphere projection away from the Schwarzschild solution, shown in blue. We will not worry about this spurious effect for the moment (as it concerns only few data points) and postpone a more thorough discussion of it to Sec. $\mathrm{VA}$.

Let us also briefly comment about the features of the resulting image. In the lower panel of Fig. 1, we show the relative difference between the images of the upper panel of Fig. 1. Since the injected metric deformation [Eq. (22)] has a local minimum around $r \approx 7 M_{\mathrm{BH}}$, one could naively expect that the absolute value of the relative difference should also have a local maximum there. However, a more involved structure is evident. The 
appearance of a hill and a trough in the intensity is mostly due to the derivatives of the metric deviation from Schwarzschild becoming larger and dominant at the location of the bump - indeed, this pattern resembles the shape of the derivative of a Gaussian function.

In Fig. 3 , in blue, we show the $2 \sigma$ reconstruction contours for the deviation of the metric from Schwarzschild. As before, we observe generally good agreement with the injection (gray dashed lines) for the $N_{\text {th }}=1$ PCA criterion. The reconstructed bump is clearly distinguishable from the power law component. The narrower error bands near the photon sphere at $r \approx 3 M_{\mathrm{BH}}$ indicate greater sensitivity of the method to features in that region. As in the previous example, the goodness of the reconstruction deteriorates near the $\mathrm{BH}$ horizon $\left(r \approx 2 M_{\mathrm{BH}}\right.$ ) presumably due to the effect of gravitational redshift. Finally, we observe a slight oscillatory behavior around $r \approx 11 M_{\mathrm{BH}}$. This happens because many of the eigenvectors (and consequently the eigenfunctions) of the Fisher matrix present oscillatory features. By including more components (i.e. lower $N_{\text {th }}$ or smaller measurement error), these oscillatory components interfere and cancel out to give a better overall reconstruction.

\section{Reconstructing Astrophysics and Geometry}

Finally, we will now present the most interesting results of our framework. These correspond to cases in which both the spacetime and accretion flow are reconstructed at the same time -i.e. all of the parameters $\boldsymbol{\alpha}$ are allowed to vary. Since both aspects play an important role in the details of $\mathrm{BH}$ images, one might argue that it is not possible to constrain deviations in the metric when the exact details of the astrophysical model are not known -see for example Gralla [27]. In this section, we will show that if our "theoretical priors" are strong enough, it is in principle possible to disentangle the two types of contributions. Such strong priors can be achieved by sufficient understanding of the underlying astrophysics and by reasonable constraints on the long distance behavior of the metric.

For this example, the injection and reference functions are the same as in Sec. IV B. In this case, as expected, we find that there are degeneracies between the metric and the emissivity, when one considers a Gaussian basis for both functions. Indeed, one generically obtains degenerate reconstructions - i.e. profiles for the metric and emissivity that reproduce the data but do not correspond to the injection. Since these are "failed" reconstructions, we do not present them here.

In order to tighten our priors and mitigate this degeneracy, we assume that the radial distribution of the emissivity is well described by a power law (as in RIAF models), and thus allow only the amplitude and exponent to vary. Moreover, we linearize the emissivity in the deviation of the exponent away from the default reference value [which is set as in Eq. (22) for this example], so as

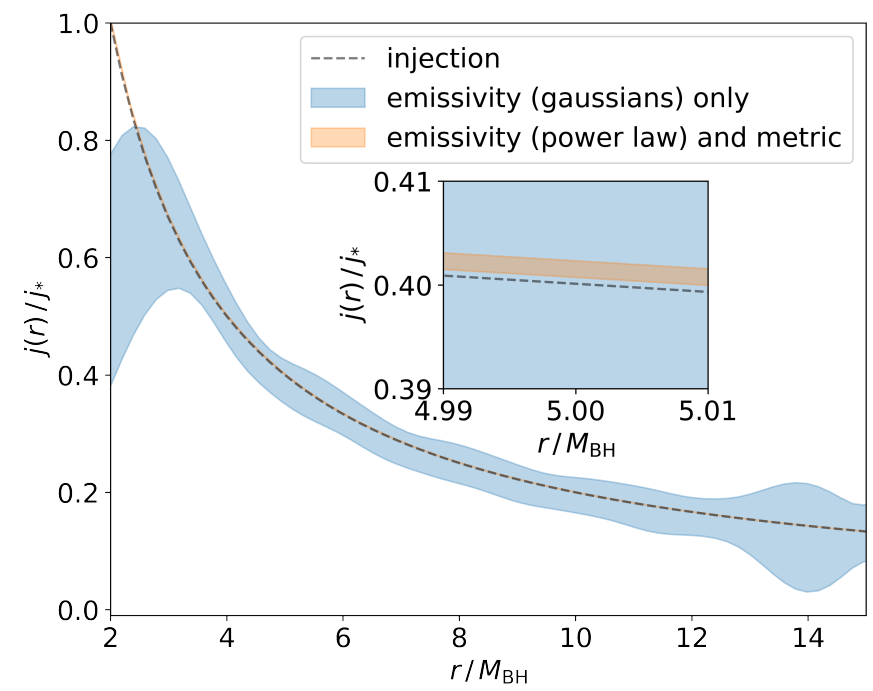

FIG. 2. Individual reconstruction of the emissivity when the metric is fixed (blue), and joint reconstruction (orange) when it is constrained to be a power law, corresponding respectively to the examples of Sec. IV A and IV C. The scale $j_{*}$ is used to normalize the emissivity and its units are given in the main text.

to reduce to Eq. (7). We take the metric basis and priors to be the same as in the previous example, and perform the reconstruction with a PCA criterion $N_{\text {th }}=1$ as before.

In Fig. 2, we show the $2 \sigma$ reconstruction contours of the emissivity in orange. Because of the tight "theoretical priors" that we assumed on the functional form of the the emissivity, the reconstruction is much better than the "agnostic" one. However, upon closer inspection, as can be seen from the inset, a slight bias is noticeable in the reconstruction. Part of it is due to the missing information weeded out by the PCA criterion. We have verified this by comparing reconstructions with different values of $N_{\text {th }}$.

In Fig. 3, one can observe that the reconstruction of the metric deviation (in orange) recovers the injection as in the previous example. It also exhibits the narrower contour bands near $r \approx 3 M_{\mathrm{BH}}$, and the slightly inaccurate reconstruction near the $\mathrm{BH}$ horizon at $r \approx 2 M_{\mathrm{BH}}$, like before.

This example illustrates that a good, but not necessarily complete, understanding of the astrophysics may be sufficient to extract possible deviations from GR from the $\mathrm{BH}$ image. Although constraining the emissivity to follow a particular parametrization - here a power law - may seem rather restrictive, this is not necessarily the only way to mitigate the degeneracy. Alternative possibilities may include judiciously prescribing the priors of the astrophysical parameters -in the spirit of the metric prior of Eq. (20)-, or reducing the number of parameters in the metric by employing a particular ansatz - e.g. the Rezzolla-Zhidenko parametrization [51]. This second possibility, however, comes at the cost of being less 


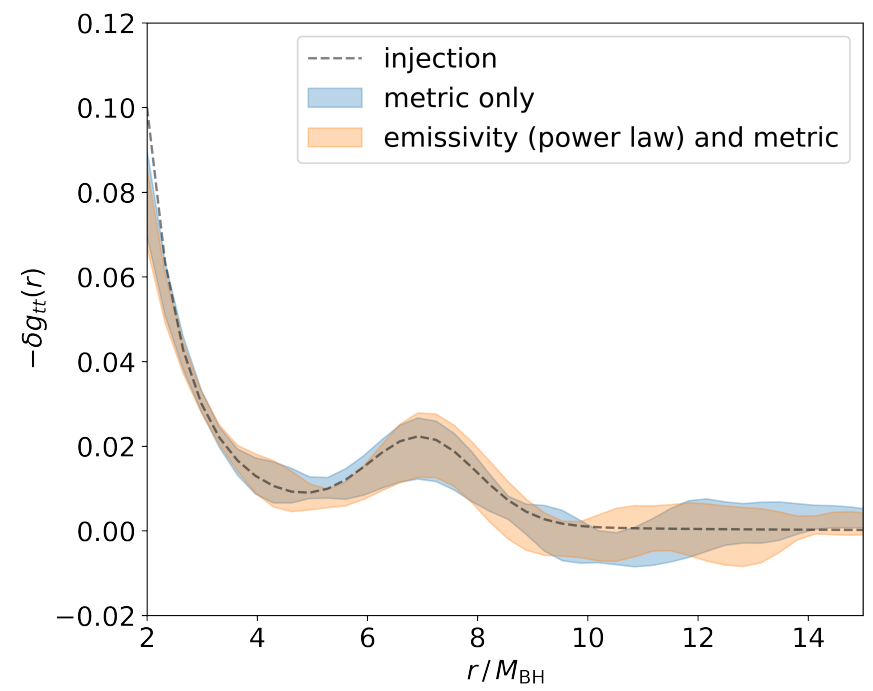

FIG. 3. Individual reconstruction of the metric deviation from Schwarzschild, $\delta g_{t t}(r)=g_{t t}(r)-g_{t t}^{(0)}(r)$, when the emissivity is fixed (blue) and joint reconstruction (orange), corresponding respectively to the examples of Sec. IV A and IV C.

general, however. We leave these possibilities to future extensions of this work.

\section{DISCUSSION}

In this Section, we briefly comment about the relation between our framework and other existing approaches (Sec. VA). Some limitations and possible extensions of this work are discussed in Sec. V B. Finally, possible connections to the inverse problem in gravitational wave astronomy are mentioned in Sec. V C.

\section{A. Comparison to Other Approaches}

Many previous works on modified gravity constraints using the EHT measurements utilize only the size of the critical curve corresponding to the projection at infinity of the photon sphere [12-25]. This is justified, because it has been argued that the size of the bright ring is a robust feature in the $\mathrm{BH}$ image $[26,29,36]$. However, whether the approach is to constrain the charges and coupling constants of particular solutions in modified theories of gravity (e.g. "exotic" Reissner-Nordström solutions $[61,62]$ ), or to constrain the parameters in phenomenological parametrizations of the metric (e.g. the Rezzolla-Zhidenko parametrization [51]), the bounds that are obtained are generically degenerate and loose when many parameters are at play [28]. This is not surprising since the shadow size amounts to the measurement of a single number. Indeed, if our PCA framework is applied to the shadow size alone (see Appendix A), the only combination of parameters that can be constrained [c.f. Eq. (11) of Ref. [28]] is

$$
\sum_{i=1}^{M} \alpha_{i} \delta g_{t t}^{(i)}\left(3 M_{\mathrm{BH}}\right)
$$

In this work, we have on the one hand exploited the whole intensity profile of the EHT BH image (although in a toy model and with mock data). On the other hand, to allow for generic deviations away from GR BHs, we have parametrized the metric in terms of a completely general superposition of basis functions. We have then performed a PCA analysis to obtain a smooth metric reconstruction, filtering out the components that cannot be significantly constrained. This technique has been applied before to describe the shape of the shadow of spinning BHs in non-Kerr metrics in Ref. [24]. However, unlike in previous applications, here it is used to reconstruct the spacetime geometry and the disk's emissivity using the whole intensity profile of the $\mathrm{BH}$ image.

\section{B. Limitations and possible extensions}

Although astrophysical $\mathrm{BHs}$ are believed to be simple (i.e. describable only by their mass and angular momentum [8-10, 63]), the modelling of their environments is fairly complex. In this work, we have made a large number of simplifications (no spin, static and spherical accretion flow, etc.). Nevertheless, the inclusion of many of these environmental aspects is conceptually straightforward, although it would lead to computationally more intensive analyses.

We have made further simplifications regarding the observational data. In particular, we have assumed that the intensity profile is measured directly and that the associated errors are Gaussian and uncorrelated. The inclusion of visibilities - the observables that the EHT actually measures and which correspond to the Fourier transform coefficients of the image - is also possible in future extensions of this work. However, one would have to deal with additional errors due to the incomplete observational coverage of the visibility parameter space see e.g. Fig. 2 of Ref. [6].

Regarding the actual calculation of the $\mathrm{BH}$ image, we have linearized the radiative transfer and geodesic equations [Eqs. (5) and (3)]. This step was essential in order to make the likelihood a Gaussian function and to allow us to apply the PCA technique (which relies on the Fisher matrix being constant). For the case of the metric, this is justified by the expectation that deviations from the GR solution are small. Similarly, the linearization is reasonable for the accretion parameters, provided that one has good prior knowledge of the astrophysical model.

Even in this case, however, some non-perturbative effects may be important. In particular, the linearization does not capture the shift in the position of the photon sphere or the BH horizon. Future work in this direction may include integration of the full non-linear equa- 
tions and reconstruction with Markov Chain Monte Carlo (MCMC) techniques, along the line of Refs. [40, 64].

Finally, we also recall that we have considered a metric ansatz [Eq. (1)] satisfying $g_{t t}(r) g_{r r}(r)=-1$. It is well known that this condition does not hold in general [65]. Moreover, it is expected that BHs have spin - e.g. to power the jet seen in M87* [36, 45]. Therefore, a more rigorous analysis should necessarily include more than one independent metric function, leading to further degeneracy between them. A first step in this direction would be to consider the slowly rotating case, by assuming a fixed form of the mixed terms in the metric, i.e. $g_{t \phi}(r)=g_{\phi t}(r)=-M_{\mathrm{BH}}^{2} a / r$, and linearizing Eqs. (3) and (5) in the dimensionless spin parameter $a$. In this case (even if the accretion flow is spherical) the radial symmetry of the $\mathrm{BH}$ image is broken and we expect that the angle of observation will become important in our ability to extract $a$ with our PCA approach. In the most general case, once rotation and realistic accretion are considered, we expect that a good modelling of the astrophysical component, as well as appropriate priors that reflect our knowledge in the weak gravity regime, will continue to be key in allowing to extract possible deviations of the spacetime geometry. Moreover, although more information in the $\mathrm{BH}$ image could potentially be extracted, additional strategies may be needed handle degeneracy in the parameter space. We leave this more challenging setup for future work.

\section{Possible Connections to Gravitational Waves}

It is well known in the literature that $\mathrm{BH}$ quasi-normal modes in the eikonal approximation are closely related to the impact parameter of the $\mathrm{BH}$ shadow [66, 67]. An interesting question is therefore whether there is a gravitational wave analog to the $\mathrm{BH}$ image. Unlike the image, which depends strongly on the surrounding matter, gravitational waves couple only very weakly to the environment, which would allow for a much cleaner test of $\mathrm{BH}$ geometries.

One possibility would be to use the full, infinite set of BH quasi-normal modes [68-70]. However, the eikonal approximation only probes the spacetime near the maximum of the potential. Moreover, in general one cannot expect that the inverse spectrum problem is uniquely solvable, e.g., in GR axial and polar perturbations are isospectral, but the underlying Regge-Wheeler and Zerilli potentials are not the same (and other isospectral, and thus equivalent, potentials can be built with suitable transformations [71, 72]). While degeneracies remain, however, it is possible to put some constraints on the $\mathrm{BH}$ metric or the potential for quasi-normal modes, given a finite set of quasi-normal mode measurements (see e.g. [73, 74]).

Closely related to the quasi-normal mode spectrum are the transmission and reflection coefficients, which describe the frequency dependent wave propa- gation in the spacetime. These coefficients are computed over the whole exterior $\mathrm{BH}$ spacetime. In the Wentzel-Kramers-Brillouin (WKB) approximation [75] this can be done via integral equations, which one can attempt to invert to constrain the properties of the potentials [76-78]. This can also be connected to Hawking radiation [79]. Like for the quasi-normal modes, however, the problem is not uniquely invertible.

\section{CONCLUSIONS}

In this work, we have demonstrated that $\mathrm{BH}$ spacetimes and simple accretion models can be constrained at the same time from $\mathrm{BH}$ images. The general inverse problem of $\mathrm{BH}$ imaging involves the reconstruction of the astrophysical properties of the accretion disk, as well as possible deviations of the spacetime geometry from GR. Our analysis is a proof of principle, because it is only valid (strictly speaking) for spherically symmetric spacetimes and it makes considerable simplifications for the accretion model and for the mock data. It is clear that realistic modelling of astrophysical processes, as well as of the EHT data analysis pipeline, exceed what can be described by our simple current framework. However, the detailed study of a simplified toy models is still of great value and interest, since it allows one to understand the fundamental aspects of the problem. This is also well demonstrated by the ongoing discussion about the interpretation of the bright emission ring observed by the EHT collaboration, and namely about whether that can be robustly identified with the impact parameter of the photon orbit, and thus be used to test the Kerr hypothesis [26-30].

In summary, our framework addresses the fundamental question of whether astrophysical uncertainties and degeneracies jeopardize tests of the Kerr hypothesis with EHT BH images. By deriving a linear model that describes the BH geometry in terms of a general superposition of basis functions (Gaussians and power laws), we have demonstrated that a PCA technique allows for reconstructing the spacetime geometry and the accretion model simultaneously, provided that sufficient theoretical priors are available on the latter.

\section{ACKNOWLEDGMENTS}

We thank Avery E. Broderick and Boris Georgiev for useful comments on accretion disk models in the earlier stages of this work, as well as Luciano Rezzolla and Maciek Wielgus for insightful comments on this manuscript. All authors acknowledge financial support provided under the European Union's H2020 ERC Consolidator Grant "GRavity from Astrophysical to Microscopic Scales" grant agreement no. GRAMS-815673. This work was supported by the EU Horizon 2020 
Research and Innovation Programme under the Marie Skłodowska-Curie Grant Agreement No. 101007855.

\section{Appendix A: Black hole shadow with PCA}

In this appendix we apply the PCA framework to the shadow size of a non-rotating $\mathrm{BH}$ and conclude that only one combination of parameters can be meaningfully constrained. We will ignore the effect of spin as in Ref. [29].

The impact parameter $b_{\mathrm{ph}}$ and radial location $r_{\mathrm{ph}}$ of the photon sphere are obtained by solving the system $V\left(r_{\mathrm{ph}}, b_{\mathrm{ph}}\right)=\partial V\left(r_{\mathrm{ph}}, b_{\mathrm{ph}}\right) / \partial r=0$, where $V(r, b)$ is the effective potential of null rays in spherical symmetry. Remarkably, $V(r)$ is only sensitive to the $g_{t t}(r)$ metric function, and therefore, no constraints can be placed on an independent function $g_{r r}(r)$. Explicitly, this system becomes [28]

$$
r_{\mathrm{ph}}=\frac{2 g_{t t}\left(r_{\mathrm{ph}}\right)}{g_{t t}^{\prime}\left(r_{\mathrm{ph}}\right)}, \quad b_{\mathrm{ph}}^{2}=-\frac{4 g_{t t}\left(r_{\mathrm{ph}}\right)}{\left[g_{t t}^{\prime}\left(r_{\mathrm{ph}}\right)\right]^{2}} .
$$

For the Schwarzschild metric, these equations can be easily solved to obtain $r_{\mathrm{ph}}^{\mathrm{Sch}}=3 M_{\mathrm{BH}}$ and $b_{\mathrm{ph}}^{\mathrm{Sch}}=3 \sqrt{3} M_{\mathrm{BH}}$.

A deformed Schwarzschild metric introduces deviations on the photon sphere radius $\delta r_{\mathrm{ph}}$ and impact parameter $\delta b_{\mathrm{ph}}$. By linearizing Eqs. (A1), they are found to be

$$
\begin{aligned}
\delta r_{\mathrm{ph}} & =-\frac{3}{8}\left[3 r_{s}^{2} \delta g_{t t}^{\prime}\left(r_{\mathrm{ph}}^{\mathrm{Sch}}\right)-4 r_{s} \delta g_{t t}\left(r_{\mathrm{ph}}^{\mathrm{Sch}}\right)\right], \\
\delta b_{\mathrm{ph}} & =\frac{9}{4} \sqrt{3} r_{s} \delta g_{t t}\left(r_{\mathrm{ph}}^{\mathrm{Sch}}\right),
\end{aligned}
$$

where $r_{s}=2 M_{\mathrm{BH}}$ and where we can describe $\delta g_{t t}(r)$ as a sum of $M$ basis terms with parameters $\alpha_{i}$ as in Eq. (6) in the main text. Our model for the impact parameter - the only observable- will then be $b_{M}(\boldsymbol{\alpha})=b_{\mathrm{ph}}^{\mathrm{Sch}}+\delta b_{\mathrm{ph}}(\boldsymbol{\alpha})$.

We compare with a measurement $b_{\mathrm{ph}}^{\mathrm{Sch}} \pm \sigma$, with error $\sigma \approx 0.17 b_{\mathrm{ph}}^{\mathrm{Sch}}[26]$, by writing the likelihood

$$
\log p\left(\boldsymbol{\alpha} \mid b_{\mathrm{ph}}^{\mathrm{Sch}}\right)=-\frac{\left(b_{M}(\boldsymbol{\alpha})-b_{\mathrm{ph}}^{\mathrm{Sch}}\right)^{2}}{2 \sigma^{2}} .
$$

For flat priors, the Fisher matrix (13) becomes

$$
F_{i j} \propto \delta g_{t t}^{(i)}\left(r_{\mathrm{ph}}^{\mathrm{Sch}}\right) \delta g_{t t}^{(j)}\left(r_{\mathrm{ph}}^{\mathrm{Sch}}\right)
$$

which has only one non-zero eigenvalue, corresponding to the eigenvector

$$
\left.\boldsymbol{e}^{(1)} \propto\left(\delta g_{t t}^{(1)}, \ldots, \delta g_{t t}^{(M)}\right)\right|_{r_{\mathrm{ph}}^{\mathrm{Sch}}}
$$

Then, the only combination [c.f. Eq. (11) of Ref. [28]] we can constrain with the EHT measurement is

$$
\left|\sum_{i=1}^{M} \alpha_{i} \delta g_{t t}^{(i)}\left(r_{\mathrm{ph}}^{\mathrm{Sch}}\right)\right| \lesssim 0.17 .
$$

[1] A. Einstein, The Foundation of the General Theory of Relativity, Annalen Phys. 49, 769 (1916).

[2] B. P. Abbott and et al. (LIGO Scientific Collaboration and Virgo Collaboration), Observation of gravitational waves from a binary black hole merger, Phys. Rev. Lett. 116, 061102 (2016).

[3] B. P. Abbott and et al. (LIGO Scientific Collaboration and Virgo Collaboration), Gw170817: Observation of gravitational waves from a binary neutron star inspiral, Phys. Rev. Lett. 119, 161101 (2017).

[4] B. P. Abbott and et al. (LIGO Scientific and Virgo Collaborations), Tests of general relativity with gw150914, Phys. Rev. Lett. 116, 221101 (2016).

[5] R. Abbott et al. (LIGO Scientific, Virgo), Tests of general relativity with binary black holes from the second LIGOVirgo gravitational-wave transient catalog, Phys. Rev. D 103, 122002 (2021), arXiv:2010.14529 [gr-qc].

[6] K. Akiyama et al. (Event Horizon Telescope), First M87 Event Horizon Telescope Results. I. The Shadow of the Supermassive Black Hole, Astrophys. J. 875, L1 (2019), arXiv:1906.11238 [astro-ph.GA].

[7] K. Akiyama et al. (Event Horizon Telescope), First M87 Event Horizon Telescope Results. VI. The Shadow and Mass of the Central Black Hole, Astrophys. J. Lett. 875, L6 (2019), arXiv:1906.11243 [astro-ph.GA].
[8] D. Robinson, Uniqueness of the Kerr black hole, Phys. Rev. Lett. 34, 905 (1975).

[9] W. Israel, Event horizons in static vacuum space-times, Phys. Rev. 164, 1776 (1967).

[10] S. Hawking, Black holes in general relativity, Commun. Math. Phys. 25, 152 (1972).

[11] R. P. Kerr, Gravitational field of a spinning mass as an example of algebraically special metrics, Phys. Rev. Lett. 11, 237 (1963).

[12] R. Takahashi, Black hole shadows of charged spinning black holes, Publ. Astron. Soc. Jap. 57, 273 (2005), arXiv:astro-ph/0505316.

[13] T. Johannsen and D. Psaltis, Testing the No-Hair Theorem with Observations in the Electromagnetic Spectrum: II. Black-Hole Images, Astrophys. J. 718, 446 (2010), arXiv:1005.1931 [astro-ph.HE].

[14] D. Psaltis and T. Johannsen, Sgr A*: The Optimal Testbed of Strong-Field Gravity, J. Phys. Conf. Ser. 283, 012030 (2011), arXiv:1012.1602 [astro-ph.HE].

[15] L. Amarilla and E. F. Eiroa, Shadow of a rotating braneworld black hole, Phys. Rev. D 85, 064019 (2012), arXiv:1112.6349 [gr-qc].

[16] A. E. Broderick, T. Johannsen, A. Loeb, and D. Psaltis, Testing the No-Hair Theorem with Event Horizon Telescope Observations of Sagittarius A*, Astrophys. J. 784, 
7 (2014), arXiv:1311.5564 astro-ph.HE].

[17] D. Psaltis, F. Ozel, C.-K. Chan, and D. P. Marrone, A General Relativistic Null Hypothesis Test with Event Horizon Telescope Observations of the blackhole shadow in Sgr A*, Astrophys. J. 814, 115 (2015), arXiv:1411.1454 [astro-ph.HE].

[18] T. Johannsen, A. E. Broderick, P. M. Plewa, S. Chatzopoulos, S. S. Doeleman, F. Eisenhauer, V. L. Fish, R. Genzel, O. Gerhard, and M. D. Johnson, Testing General Relativity with the Shadow Size of Sgr A*, Phys. Rev. Lett. 116, 031101 (2016), arXiv:1512.02640 [astroph.GA].

[19] D. Psaltis, N. Wex, and M. Kramer, A Quantitative Test of the No-Hair Theorem with Sgr A* using stars, pulsars, and the Event Horizon Telescope, Astrophys. J. 818, 121 (2016), arXiv:1510.00394 [astro-ph.HE].

[20] P. V. P. Cunha, C. A. R. Herdeiro, E. Radu, and H. F. Runarsson, Shadows of Kerr black holes with scalar hair, Phys. Rev. Lett. 115, 211102 (2015), arXiv:1509.00021 [gr-qc].

[21] P. V. Cunha, C. A. R. Herdeiro, B. Kleihaus, J. Kunz, and E. Radu, Shadows of Einstein-dilaton-Gauss-Bonnet black holes, Phys. Lett. B 768, 373 (2017), arXiv:1701.00079 [gr-qc].

[22] D. Psaltis, Testing General Relativity with the Event Horizon Telescope, Gen. Rel. Grav. 51, 137 (2019), arXiv:1806.09740 [astro-ph.HE].

[23] P. V. Cunha, C. A. Herdeiro, and E. Radu, Spontaneously Scalarized Kerr Black Holes in Extended ScalarTensor-Gauss-Bonnet Gravity, Phys. Rev. Lett. 123, 011101 (2019), arXiv:1904.09997 [gr-qc].

[24] L. Medeiros, D. Psaltis, and F. Özel, A Parametric model for the shapes of black-hole shadows in non-Kerr spacetimes, Astrophys. J. 896, 7 (2020), arXiv:1907.12575 [astro-ph.HE].

[25] Z. Younsi, A. Zhidenko, L. Rezzolla, R. Konoplya, and Y. Mizuno, New method for shadow calculations: Application to parametrized axisymmetric black holes, Phys. Rev. D 94, 084025 (2016), arXiv:1607.05767 [gr-qc].

[26] D. Psaltis et al. (EHT Collaboration), Gravitational Test beyond the First Post-Newtonian Order with the Shadow of the M87 Black Hole, Phys. Rev. Lett. 125, 141104 (2020).

[27] S. E. Gralla, Can the EHT M87 results be used to test general relativity?, Phys. Rev. D 103, 024023 (2021), arXiv:2010.08557 [astro-ph.HE].

[28] S. H. Völkel, E. Barausse, N. Franchini, and A. E. Broderick, EHT tests of the strong-field regime of General Relativity, Class. Quant. Grav. 38, 21 (2020), arXiv:2011.06812 [gr-qc].

[29] P. Kocherlakota et al. (Event Horizon Telescope), Constraints on black-hole charges with the 2017 EHT observations of M87*, Phys. Rev. D 103, 104047 (2021), arXiv:2105.09343 [gr-qc].

[30] K. Glampedakis and G. Pappas, Can supermassive black hole shadows test the Kerr metric?, Phys. Rev. D 104 , L081503 (2021), arXiv:2102.13573 [gr-qc].

[31] S. Nampalliwar and S. Kumar, Theory-agnostic tests of gravity with black hole shadows, (2021), arXiv:2108.01190 [gr-qc].

[32] S. E. Gralla, A. Lupsasca, and D. P. Marrone, The shape of the black hole photon ring: A precise test of strongfield general relativity, Phys. Rev. D 102, 124004 (2020).
[33] A. E. Broderick, P. Tiede, D. W. Pesce, and R. Gold, Measuring Spin from Relative Photon Ring Sizes, (2021), arXiv:2105.09962 [astro-ph.HE].

[34] M. Wielgus, Photon rings of spherically symmetric black holes and robust tests of non-Kerr metrics, (2021), arXiv:2109.10840 [gr-qc].

[35] H. C. D. Lima, Junior., L. C. B. Crispino, P. V. P. Cunha, and C. A. R. Herdeiro, Can different black holes cast the same shadow?, Phys. Rev. D 103, 084040 (2021), arXiv:2102.07034 [gr-qc].

[36] K. Akiyama et al. (Event Horizon Telescope), First M87 Event Horizon Telescope Results. V. Physical Origin of the Asymmetric Ring, Astrophys. J. Lett. 875, L5 (2019), arXiv:1906.11242 [astro-ph.GA].

[37] F. H. Vincent, M. Wielgus, M. A. Abramowicz, E. Gourgoulhon, J. P. Lasota, T. Paumard, and G. Perrin, Geometric modeling of $\mathrm{M} 87^{*}$ as a Kerr black hole or a non-Kerr compact object, Astron. Astrophys. 646, A37 (2021), arXiv:2002.09226 [gr-qc].

[38] Y. Mizuno, Z. Younsi, C. M. Fromm, O. Porth, M. De Laurentis, H. Olivares, H. Falcke, M. Kramer, and L. Rezzolla, The Current Ability to Test Theories of Gravity with Black Hole Shadows, Nat. Astron. 2, 585 (2018), arXiv:1804.05812 [astro-ph.GA].

[39] L. R. Weih, H. Olivares, and L. Rezzolla, Two-moment scheme for general-relativistic radiation hydrodynamics: a systematic description and new applications, Mon. Not. Roy. Astron. Soc. 495, 2285 (2020), arXiv:2003.13580 $[\mathrm{gr}-\mathrm{qc}]$.

[40] A. E. Broderick, T. Johannsen, A. Loeb, and D. Psaltis, Testing the no-hair theorem with event horizon telescope observations of sagittarius a*, The Astrophysical Journal 784, 7 (2014).

[41] J. C. Algaba et al. (Event Horizon Telescope, FermiLAT, H.E.S.S., MAGIC, VERITAS, EAVN), Broadband Multi-wavelength Properties of M87 during the 2017 Event Horizon Telescope Campaign, Astrophys. J. Lett. 911, L11 (2021), arXiv:2104.06855 [astro-ph.HE].

[42] J. L. Walsh, A. J. Barth, L. C. Ho, and M. Sarzi, The M87 Black Hole Mass from Gas-dynamical Models of Space Telescope Imaging Spectrograph Observations, Astrophys. J. 770, 86 (2013), arXiv:1304.7273 [astro-ph.CO].

[43] K. Gebhardt, J. Adams, D. Richstone, T. R. Lauer, S. M. Faber, K. Gültekin, J. Murphy, and S. Tremaine, The Black Hole Mass in M87 from Gemini/NIFS Adaptive Optics Observations, Astrophys. J. 729, 119 (2011), arXiv:1101.1954 [astro-ph.CO].

[44] Black Holes (Les Astres Occlus), Les Houches Summer School, Vol. 23 (Gordon and Breach, New York, NY, 1973).

[45] B. Jeter and A. E. Broderick, Reconciling EHT and Gas Dynamics Measurements in M87: Is the Jet Misaligned at Parsec Scales?, Astrophys. J. 908, 139 (2021), arXiv:2010.11303 [astro-ph.GA].

[46] G. B. Rybicki and A. P. Lightman, Radiative Processes in Astrophysics (Wiley-VCH, Weinheim, 1986).

[47] A. E. Broderick, V. L. Fish, S. S. Doeleman, and A. Loeb, Evidence for Low Black Hole Spin and Physically Motivated Accretion Models from Millimeter VLBI Observations of Sagittarius A*, Astrophys. J. 735, 110 (2011), arXiv:1011.2770 [astro-ph.HE].

[48] Z. Younsi, K. Wu, and S. V. Fuerst, General relativistic radiative transfer: formulation and emission from structured tori around black holes, Astronomy \& Astrophysics 
545, A13 (2012).

[49] T. Bronzwaer, J. Davelaar, Z. Younsi, M. Mościbrodzka, H. Falcke, M. Kramer, and L. Rezzolla, RAPTOR I: Time-dependent radiative transfer in arbitrary spacetimes, Astron. Astrophys. 613, A2 (2018), arXiv:1801.10452 [astro-ph.HE].

[50] S. E. Gralla, D. E. Holz, and R. M. Wald, Black Hole Shadows, Photon Rings, and Lensing Rings, Phys. Rev. D 100, 024018 (2019), arXiv:1906.00873 [astro-ph.HE].

[51] L. Rezzolla and A. Zhidenko, New parametrization for spherically symmetric black holes in metric theories of gravity, Phys. Rev. D 90, 084009 (2014).

[52] R. Konoplya, L. Rezzolla, and A. Zhidenko, General parametrization of axisymmetric black holes in metric theories of gravity, Phys. Rev. D 93, 064015 (2016), arXiv: 1602.02378 [gr-qc].

[53] R. A. Konoplya and A. Zhidenko, General parametrization of black holes: The only parameters that matter, Phys. Rev. D 101, 124004 (2020), arXiv:2001.06100 [grqc].

[54] N. J. Cornish, T. B. Littenberg, B. Bécsy, K. Chatziioannou, J. A. Clark, S. Ghonge, and M. Millhouse, Bayeswave analysis pipeline in the era of gravitational wave observations, Physical Review D 103, 10.1103/physrevd.103.044006 (2021).

[55] S. E. Gralla, Second Order Gravitational Self Force, Phys. Rev. D 85, 124011 (2012), arXiv:1203.3189 [gr-qc].

[56] W. H. Press, S. A. Teukolsky, W. T. Vetterling, and B. P. Flannery, Numerical Recipes in C (2nd Ed.): The Art of Scientific Computing (Cambridge University Press, USA, 1992).

[57] M. Pieroni and E. Barausse, Foreground cleaning and template-free stochastic background extraction for LISA, JCAP 07, 021, [Erratum: JCAP 09, E01 (2020)], arXiv:2004.01135 [astro-ph.CO].

[58] H. O. Silva, J. Sakstein, L. Gualtieri, T. P. Sotiriou, and E. Berti, Spontaneous scalarization of black holes and compact stars from a Gauss-Bonnet coupling, Phys. Rev. Lett. 120, 131104 (2018), arXiv:1711.02080 [gr-qc].

[59] C. L. Rodriguez, B. Farr, V. Raymond, W. M. Farr, T. B. Littenberg, D. Fazi, and V. Kalogera, Basic Parameter Estimation of Binary Neutron Star Systems by the Advanced LIGO/Virgo Network, Astrophys. J. 784, 119 (2014), arXiv:1309.3273 [astro-ph.HE].

[60] M. D. Johnson, A. Lupsasca, A. Strominger, G. N. Wong, S. Hadar, D. Kapec, R. Narayan, A. Chael, C. F. Gammie, P. Galison, and et al., Universal interferometric signatures of a black hole's photon ring, Science Advances 6, eaaz1310 (2020).

[61] D. Garfinkle, G. T. Horowitz, and A. Strominger, Charged black holes in string theory, Phys. Rev. D 43 , 3140 (1991), [Erratum: Phys.Rev.D 45, 3888 (1992)].

[62] G. Gibbons and K.-i. Maeda, Black Holes and Mem- branes in Higher Dimensional Theories with Dilaton Fields, Nucl. Phys. B 298, 741 (1988).

[63] B. Carter, Axisymmetric Black Hole Has Only Two Degrees of Freedom, Phys. Rev. Lett. 26, 331 (1971).

[64] A. E. Broderick et al. (Event Horizon Telescope), THEMIS: A Parameter Estimation Framework for the Event Horizon Telescope, Astrophys. J. 897, 139 (2020).

[65] T. Jacobson, When is $\mathrm{g}(\mathrm{tt}) \mathrm{g}(\mathrm{rr})=-1$ ?, Class. Quant. Grav. 24, 5717 (2007), arXiv:0707.3222 [gr-qc].

[66] V. Ferrari and B. Mashhoon, New approach to the quasinormal modes of a black hole, Phys. Rev. D 30, 295 (1984).

[67] H. Yang, D. A. Nichols, F. Zhang, A. Zimmerman, Z. Zhang, and Y. Chen, Quasinormal-mode spectrum of Kerr black holes and its geometric interpretation, Phys. Rev. D 86, 104006 (2012), arXiv:1207.4253 [gr-qc].

[68] K. D. Kokkotas and B. G. Schmidt, Quasinormal modes of stars and black holes, Living Rev. Rel. 2, 2 (1999), arXiv:gr-qc/9909058.

[69] H.-P. Nollert, Quasinormal modes: the characteristic 'sound' of black holes and neutron stars, Classical and Quantum Gravity 16, R159 (1999).

[70] E. Berti, V. Cardoso, and A. O. Starinets, Quasinormal modes of black holes and black branes, Classical and Quantum Gravity 26, 163001 (2009).

[71] S. Chandrasekhar, The mathematical theory of black holes (Clarendon Press, Oxford, 1985).

[72] K. Glampedakis, A. D. Johnson, and D. Kennefick, Darboux transformation in black hole perturbation theory, Phys. Rev. D 96, 024036 (2017), arXiv:1702.06459 [grqc].

[73] S. H. Völkel and K. D. Kokkotas, Scalar Fields and Parametrized Spherically Symmetric Black Holes: Can one hear the shape of space-time?, Phys. Rev. D100, 044026 (2019), arXiv:1908.00252 [gr-qc].

[74] S. H. Völkel and E. Barausse, Bayesian Metric Reconstruction with Gravitational Wave Observations, Phys. Rev. D 102, 084025 (2020), arXiv:2007.02986 [gr-qc].

[75] C. M. Bender and S. A. Orszag, Advanced Mathematical Methods for Scientists and Engineers, New York: McGraw-Hill, 1978 (New York: McGraw-Hill, 1978).

[76] M. W. Cole and R. H. Good, Determination of the shape of a potential barrier from the tunneling transmission coefficient, Phys. Rev. A 18, 1085 (1978).

[77] J. C. Lazenby and D. J. Griffiths, CLASSICAL INVERSE SCATTERING IN ONE-DIMENSION, Am. J. Phys. 48, 432 (1980).

[78] S. C. Gandhi and C. J. Efthimiou, Inversion of gamow's formula and inverse scattering, American Journal of Physics 74, 638 (2006), https://doi.org/10.1119/1.2190683.

[79] S. H. Völkel, R. Konoplya, and K. D. Kokkotas, Inverse problem for Hawking radiation, Phys. Rev. D 99, 104025 (2019), arXiv:1902.07611 [gr-qc]. 